\title{
Harnessing polarization spatio-temporal coupling: a new degree of freedom in ultrafast laser material processing
}

\author{
Aabid Patel, Martynas Beresna and Peter G. Kazansky \\ Optoelectronics Research Centre, University of Southampton, SO17 1BJ, United Kingdom \\ ap7g12@orc.soton.ac.uk
}

\begin{abstract}
Polarization spatio-temporal coupling reveals new degree of freedom in ultrafast laser material processing. Control of modification in fused silica is demonstrated with the use of prism compressors and polarization azimuth of ultrashort pulse laser beam.

OCIS codes: (140.3390) Laser material processing; (320.7120) Ultrafast phenomena
\end{abstract}

The nanostructuring of transparent media with ultrafast laser pulses has attracted significant interest due to its unique applications. However, little is understood with respect to the physics of light-matter interaction. While most of the formalism for ultrashort pulses expresses the electric field separately in terms of temporal and spatial dependences, one of the major properties of ultrashort pulse generation is spatio-temporal distortions. Studies have shown spatio-temporal induced phenomena with respect to writing direction $[1,2]$ and anisotropic photosensitivity [3] all based around pulse front tilt (PFT) yet they lack the control and understanding of the orientation, direction and quantity of the spatio-temporal distortions. Here we demonstrate the control and characterization of spatio-temporal distortions and PFT with the use of prism compressors in order to control and understand ultrafast phenomena associated with material modification. We also demonstrate anisotropic photosensitivity in fused silica for the first time, to the author's knowledge, which adds an additional level of control with respect to laser material modification.

a.)

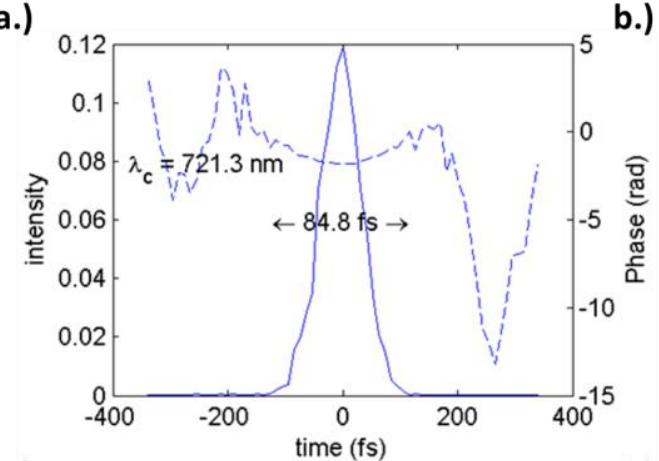

c.)

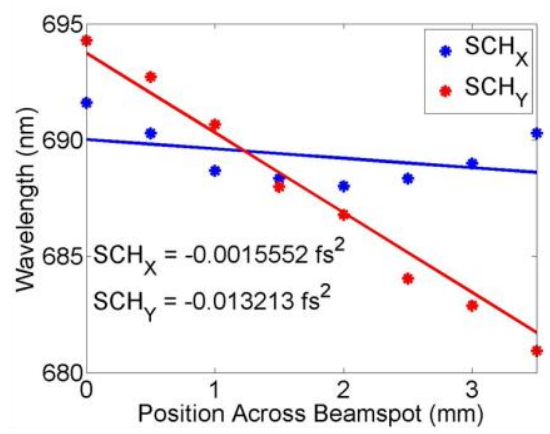

b.)

d.)
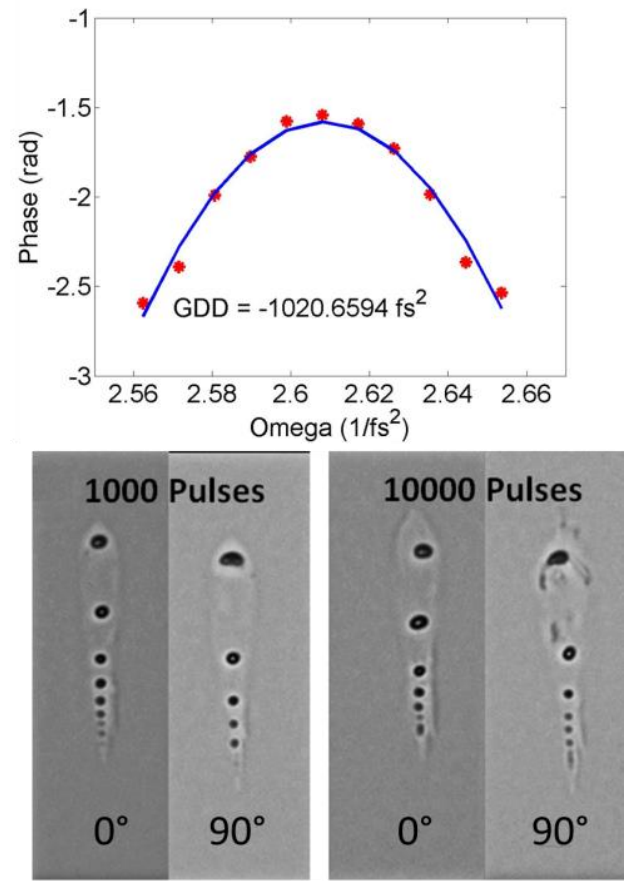

Fig. 1: a.) FROG measurement yields the electric field and pulse duration b.) GDD measurement from the retrieved phase and c.) Spatial chirp measurement in the X (blue) and Y (red), showing control of direction and orientation of PFT d.) Resulting blade effect in fused silica for 1000 and 10000 pulses when polarization is parallel $\left(0^{\circ}\right)$ and perpendicular $\left(90^{\circ}\right)$ to PFT (Pulse Energy $=1.2 \mu \mathrm{J}, \mathrm{NA}=0.65, \mathrm{PFT}=13.5 \mathrm{fs} / \mathrm{mm}$ )

A mode-locked regenerative amplified Ytterbium doped Potassium-Gadolinium Tungstate (Yb:KGW) based femtosecond PHAROS laser system (Light Conversion Ltd.) was used in conjunction with a non-collinear optical parametric amplifier (NOPA) to generate femtosecond pulses at $700 \mathrm{~nm}$ with $200 \mathrm{kHz}$ repetition rate. A prism compressor in a double pass geometry is used to control the temporal chirp and pulse duration. The laser is then sent to a stage set-up where the laser is focussed into the bulk of fused silica with a $0.65 \mathrm{NA}$ objective lens. A $\lambda / 2$ waveplate is used to control the polarization orientation with respect to the PFT. The pulses are characterized using a ThorLabs Pulse Characterization FROG Scanning Module; a SHG-based FROG. The ThorLabs based retrieval software measures the trace and reconstructs the electric field to provide the pulse duration and the group delay 
dispersion $(G D D)$ of the pulse (Fig. 1). The SCH induced from the NOPA [4] was sufficient enough to provide strong PFT and is measured by scanning a spectrometer across the pulse with the slope of the wavelength vs. position (Fig. 1). Additional SCH can be added and controlled with the use of another set of prisms in a single pass geometry. With control of the GDD and constant SCH, the PFT can be controlled accordingly:

$$
P F T=A D+(S C H * T C H)[5]
$$

Ensuring the $\mathrm{AD}$ is negligible, $\mathrm{PFT}$ is dominated and controlled with the respective chirps.

Single spot modification experiments in fused silica are done at multiple exposures at 85 fs and $1.2 \mu \mathrm{J}$ (Fig. 1). PFT in this case is $13.5 \mathrm{fs} / \mathrm{mm}$ before the objective lens. After the objective lens, the PFT increases to $43.94 \mathrm{fs} / \mathrm{mm}$ due to the GDD induced by the glass in the lens before focusing; a proponent of spatio-temporal distortions that has been overlooked by the community. While rotating the polarization state with respect to the PFT, there is a clear difference in bubble formation along the modified structure. When the polarization is parallel to the PFT, the bubble formation is spread along the structure. When the polarization is perpendicular to the PFT, there is a unique bubble at the top of the structure with a clear separation between the head to the bubbles in the tail. The difference in structure confirms the anisotropy of light-matter interaction produced by the mutual orientation of polarization and pulse front tilt; known as the "blade effect" viewed in alumosilicate [3] and introduces a new level of control of modification in transparent materials that has been overlooked within the community. The blade effect is more apparent at higher pulse energies (Fig. 2).

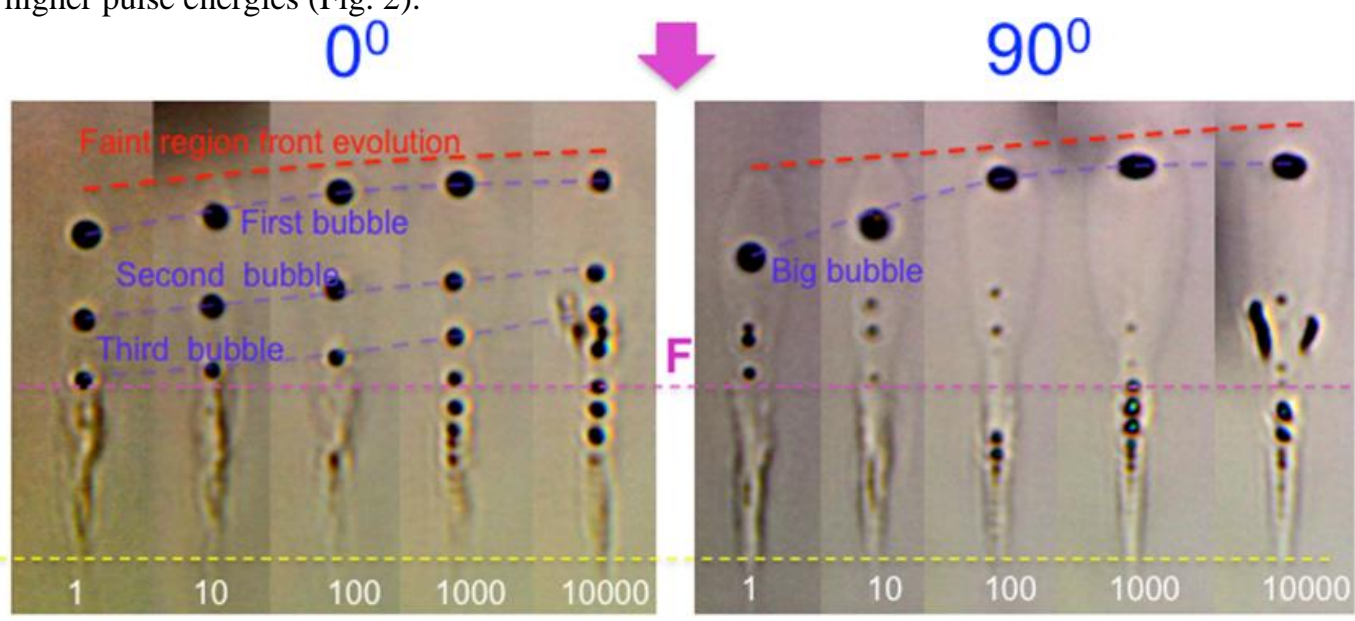

Fig 2: Single spot modification in fused silica for multiple exposures when parallel $\left(0^{\circ}\right)$ and perpendicular to PFT $\left(90^{\circ}\right)$. Modification is stronger in the $90^{\circ}$ case with modification focused at the top of the structure with larger faint region and bubble formation where in the $0^{\circ}$ case, modification is spread across the structure to the focus (F; pink dotted line) (Pulse Energy $=2.4 \mu \mathrm{J}, \mathrm{NA}=0.65, \mathrm{PFT}=3 \mathrm{fs} / \mathrm{mm}$ before objective).

The modification is different in strength with the parallel case being spread across the structure whereas in the perpendicular case, the modification is focused at the head of the structure, yielding a more focused and stronger modification. The blade effect in fused silica is surveyed in the regime of varying PFT to demonstrate the prominence of the phenomenon and the importance of spatio-temporal distortions in ultrafast laser material processing.

\section{References:}

[1] W. Yang, P. P. G. Kazansky, Y. Shimotsuma, M. Sakakura, K. Miura, and K. Hirao, "Ultrashort-pulse laser calligraphy," Appl. Phys. Lett. 93, 171109 (2008).

[2] D. Vitek, E. Block, Y. Bellouard, and D. Adams, "Spatio-temporally focused femtosecond laser pulses for nonreciprocal writing in optically transparent materials," Opt. Express 18, 24673-24678 (2010).

[3] P. G. Kazansky, Y. Shimotsuma, M. Sakakura, M. Beresna, M. Gecevičius, Y. Svirko, S. Akturk, J. Qiu, K. Miura, and K. Hirao, "Photosensitivity control of an isotropic medium through polarization of light pulses with tilted intensity front.," Opt. Express 19, 20657-64 (2011).

[4] A. Zaukevičius, V. Jukna, R. Antipenkov, V. Martinènaitè, A. Varanavičius, A. P. Piskarskas, and G. Valiulis, "Manifestation of spatial chirp in femtosecond noncollinear optical parametric chirped-pulse amplifier," JOSA B 28, 2902-2908 (2011).

[5] S. Akturk, X. Gu, P. Gabolde, and R. Trebino, "The general theory of first-order spatio-temporal distortions of Gaussian pulses and beams.," Opt. Express 13, 8642-61 (2005). 